\title{
Envelhecimento saudável e atividade física: uma revisão sistemática sobre os efeitos do exercício nas doenças cardiovasculares
}

\author{
Healthy aging and physical activity: a systematic review on the effects of exercise \\ on cardiovascular diseases
}

\author{
André Luiz Estrela ${ }^{1}$, Moisés Evandro Bauer ${ }^{1} \bowtie$ \\ ${ }^{1}$ Laboratório de Imunologia do Envelhecimento, Instituto de Pesquisas Biomédicas da Pontifícia Universidade Católica do Rio Grande do Sul. Porto Alegre, RS
}

\section{RESUMO}

Objetivos: Realizar uma revisão sistemática dos ensaios clínicos randomizados que avaliaram os benefícios do exercício físico (regular ou competitivo) para a saúde de idosos e a prevenção de doenças cardiovasculares.

Métodos: Foram investigados os registros no Pubmed, Web of Science, Library of Congress, Central de Registros Cochrane e as bases de dados com os registros dos ensaios clínicos de 2005 a 2016, juntamente com os anais das maiores conferências sobre doenças cardiovasculares no envelhecimento usando os termos definidos de acordo com a metodologia "paciente, intervenção, comparação e resultado". Os descritores utilizados foram physical effort, exercise, exercise therapy, physical activity, physical fitness, resistance training, cardiovascular disease, C-reactive protein, dyslipidemia, heart failure, cardiac insufficiency, revascularization, cardiac stent e heart transplant. Somente foram incluídos ensaios clínicos randomizados, com indivíduos acima de 60 anos de idade. Os artigos foram avaliados independentemente por dois avaliadores.

Resultados: Foram acessados um total de 3672 estudos e foram eleitos os 24 que preencheram os critérios de inclusão. A maioria desses estudos descreviam amostras reduzidas e apresentavam o exercício físico como um componente para a saúde. Apenas 16 estudos apresentavam relação da aderência à prática de exercícios físicos como prevenção cardiovascular. As medidas de resultados incluem fatores de qualidade de vida, capacidade física ou estado de condicionamento físico, força muscular, frequência cardíaca, aspectos psicológicos, marcadores inflamatórios e nutricionais e a progressão de doenças cardiovasculares. As evidências mais fortes apontam para os efeitos do exercício aeróbico na melhoria da capacidade física, força muscular e qualidade de vida de pacientes idosos com doença cardiovascular.

Conclusões: Os benefícios do treinamento físico para pacientes idosos com doença cardiovascular estão bem estabelecidos, apoiando a prescrição de exercícios físicos nos seus tratamentos regulares. Intervenções que utilizam o exercício precisam ser progressivamente incluídas nos regimes dos centros de tratamento cardiovascular, embora o melhor protocolo de exercícios para pacientes com doença cardiovascular ainda não esteja completamente estabelecido.

DESCRITORES: envelhecimento; doenças cardiovasculares; exercício aeróbico; educação física e treinamento.

\section{ABSTRACT}

Aims: To perform a systematic review of randomized controlled trials that assess the benefits of physical exercise (regular or competitive) for the health of the elderly and prevention of cardiovascular diseases.

Methods: Publications in PubMed, Web of Science, Library of Congress, Cochrane, and in databases with records of clinical trials between 2005 and 2016, and also in the proceedings of major congresses on cardiovascular diseases in the elderly were investigated using the terms defined in accordance with the "patient, intervention, comparison, and outcome" methodology. The following key words were used: physical effort, exercise, exercise therapy, physical activity, physical fitness, resistance training, cardiovascular disease, C-reactive protein, dyslipidemia, heart failure, cardiac insufficiency, revascularization, cardiac stent and heart transplant. Only randomized clinical trials with individuals aged over 60 years were included. The articles were assessed independently by two reviewers.

Results: A total of 3,672 studies were assessed and 24 that met the inclusion criteria were selected. Most of these studies had small sample sizes and showed physical exercise as health-promoting. Only 16 studies reported the relationship of adherence to physical exercise as a preventive factor against cardiovascular diseases. The outcome measures included quality of life, physical ability or fitness status, muscle strength, heart rate, psychological aspects, inflammatory and nutritional markers, and progression of cardiovascular diseases. There is strong evidence that aerobic exercise improves physical fitness, muscle strength, and the quality of life of elderly patients with cardiovascular disease. Conclusions: The benefits of exercise training for elderly patients with cardiovascular disease are well established, supporting the prescription of exercise training for their regular treatments. Interventions that use exercise need to be progressively included in cardiovascular treatment regimens, although the best exercise protocol for patients with cardiovascular disease is not yet fully established.

KEY WORDS: aging; cardiovascular diseases; exercise, aerobic; physical education and training. 
Abreviaturas: DCV, doenças cardiovasculares; ECR, ensaios clínicos randomizados; FCM, frequência cardíaca máxima; $\mathrm{TNF}$, fator de necrose tumoral; IMC, índice de massa corporal; IL, interleucina; HDL-c, colesterol ligado à lipoproteína de alta densidade; LDL-c, colesterol ligado à lipoproteína de baixa densidade; PCR, proteína C reativa; PRISMA, Preferred Reporting Items for Systematic Reviews and Meta-analyses; VFC, variabilidade de frequência cardíaca; $\mathrm{VO}_{2}$, volume de oxigênio.

\section{INTRODUÇÃO}

O envelhecimento populacional do Brasil apresenta uma estimativa de crescimento de 5,54\% até o ano de 2030 (um acréscimo de aproximadamente 11 milhões de pessoas com idade superior a 60 anos), de acordo com os dados do crescimento populacional do IBGE (2008). Nos últimos 150 anos o envelhecimento foi considerado como um processo complexo decorrente do acúmulo de múltiplas de formas de danos e patologias em diferentes tecidos como consequência da falha na manutenção celular [1].

Muitos mecanismos estão envolvidos no envelhecimento. Alguns representam degenerações naturais, tendo como principais exemplos sarcopenia, imunossenescência, diminuição de densidade mineral óssea e aumento da incidência de doenças neurodegenerativas, cardiovasculares e de câncer [2-4]. Várias intervenções podem desacelerar o processo de envelhecimento, incluindo a diminuição do tabagismo, as dietas balanceadas, os processos de hidratação e principalmente a prática regular de exercícios físicos [5-10].

A doença cardiovascular (DCV) permanece liderando as causas de morbidade e mortalidade nas sociedades modernas em todas as faixas etárias, embora a idade seja um fator de risco primário [11]. Ademais, a dieta oriunda do ocidente, rica em gordura e glicose [8,12], está diretamente associada com DCV [2,9]. As alterações vasculares relacionadas à dilatação dependente do endotélio $[11,13]$ são os principais fatores que interferem no envelhecimento [14]. Por outro lado, um ponto importante é que tanto a disfunção endotelial como a dieta inadequada pode ser parcialmente ou completamente revertida com a inclusão da população em programas de exercício e a adesão de um estilo de vida saudável $[4,14,15]$. Diversos autores apresentam as capacidades aeróbicas intrínsecas decorrentes de boa condição física como fatores determinantes na atenuação dos riscos para aterosclerose e redução da obesidade abdominal. Por outro lado, o tabagismo, a dieta não balanceada e o sedentarismo são associados ao aumento de duas a quatro vezes o risco de acidente vascular encefálico, isquêmico ou hemorrágico $[7,13,15]$, alterando diretamente o processo de envelhecimento [9].

A prática de atividade física regular, principalmente aeróbica, tornou-se um dos elementos chave na prevenção de doenças crônicas. A atividade física reduz o risco de doenças crônicas complexas, particularmente DCV isquêmica, contribui para o controle da pressão arterial e da glicemia e resulta em melhoras relacionadas com saúde e qualidade de vida. A prescrição de exercícios é bastante usual e, considerando que os níveis de atividade física entre os pacientes com DCV é significantemente menor que entre seus pares saudáveis, a baixa capacidade aeróbia pode ser melhorada com a prática regular de exercícios físicos [16]. A condição cardiorrespiratória é um potente preditor da mortalidade em pacientes com DCV $[5,9,17]$. Assumindo que a cada dia mais pacientes buscam os benefícios da prática regular de atividades físicas e aderem a um estilo de vida mais saudável, estamos presenciando um aumento real dessa população em competições, principalmente nas corridas de média e longa distância $[18,19]$. Desta maneira devemos considerar o volume do exercício como o principal componente do treinamento físico relacionado ao tratamento em qualquer estágio da doença.

Com intenção de avaliar criticamente as evidências disponíveis neste tema, realizamos uma revisão sistemática da literatura, buscando ensaios clínicos randomizados (ECR) utilizados para avaliar os benefícios do exercício físico (regular ou competitivo) na saúde de idosos e na prevenção de DCV.

\section{MÉTODOS}

O estudo foi conduzido de acordo com a diretriz para condução de metanálises de estudos de intervenção, PRISMA (Preferred Reporting Items for Systematic Reviews and Meta-analyses) [20,21]. Os critérios de elegibilidade foram estudos de ECR publicados em língua inglesa e que avaliaram a intervenção de qualquer tipo de intervenção do exercício físico, incluindo os avisos e propagandas para a prática de atividades físicas, com pacientes com DCV, independentemente do estágio, realizados com indivíduos idosos ( $\geq 60$ anos de idade). Os estudos foram baseados em amostras semelhantes, porém resultados diferenciados foram incluídos.

\section{Busca e extração dos dados}

A busca foi realizada iniciando com os artigos publicados a partir de 2005 até março de 2016 utilizando as bases de dados PubMed, Web of Science 
e Library of Congress, incluindo artigos no idioma inglês. Os resultados destas pesquisas foram agrupados com os resultados encontrados na Central de Registros Cochrane e nas bases de dados com os registros dos ensaios clínicos de 2005 a 2016. A pesquisa inicial incluiu termos como aging, exercise, physical activity e cardiovascular disease, relacionados utilizando termos associados a alta sensibilidade da estratégia de pesquisa. A maioria dos estudos elegíveis foi encontrada na base de dados PubMed.

De acordo com a especificidade da estratégia de busca da base PubMed, foram utilizados os seguintes termos: (((() Physical effort [MeSH Terms]) OU exercise [MeSH Terms]) OU 'exercise therapy' [MeSH Terms]) OU 'physical activity' [MeSH Terms]) OU 'physical fitness' [MeSH Terms]) OU 'resistance training' [MeSH Terms]) OU 'aerobic exercise' [MeSH Terms]) OU exercise [MeSH Terms] $]))$ OU ((()(((exercise) OU 'physical training') OU 'physical activity') OU (aerobic exercise') OU 'aerobic training') OU 'resistance program') OU 'resistance exercise') OU 'resistance training') OU 'aerobic program') OU 'physical activities') OU 'exercise therapy') OU 'physical tests') OU 'physical rehabilitation'). ((()((()Cardiovascular disease $[\mathrm{MeSH}$ Terms]) OU C-reactive protein [MeSH Terms]) OU dyslipidemia [MeSH Terms]) OU cardiac failure [MeSH Terms]) OU cardiac insufficiency [MeSH Terms]) OU revascularization [MeSH Terms]) OU stent cardiac [MeSH Terms]) ou heart transplant [MeSH Terms]).

Os artigos identificados foram rastreados por dois extratores diferentes com procedimento cego de resultados para o autor. A identificação inicial foi realizada somente com os títulos e resumos. Após, os textos completos dos potenciais artigos selecionados foram avaliados. A extração dos dados dos ECR selecionados foi realizada por dois revisores independentes. As discrepâncias encontradas entre os resultados encontrados pelos dois avaliadores foram discutidas até alcançar o consenso.

\section{Avaliação do risco de viés}

Os revisores independentemente acessaram os riscos de viés dos estudos incluídos utilizando a ferramenta da Colaboração Cochrane [22]. A qualidade dos ECR foi julgada pela seleção do viés (método de recrutamento, método adequado de randomização, similaridade dos grupos, critérios de elegibilidade), detecção do viés (utilização da avaliação de resultados mascarados, avaliadores e pacientes cegados) e o viés de atrito (nível de aderência à intervenção, integralidade de acompanhamento e uso de análise de intenção de tratamento); qualquer discordância em relação a extração de dados e/ou qualidade foi resolvida em uma reunião de consenso.

Cada item foi avaliado através da atribuição de um julgamento de alto ou baixo risco de viés material. O viés material foi definido como o viés de suficiente magnitude a ter efeito notável nos resultados ou conclusões, reconhecendo a subjetividade de cada julgamento.

\section{RESULTADOS DA SELEÇÃO}

$\mathrm{Na}$ busca inicial foram encontrados 3.672 artigos, sendo excluídos 547 artigos duplicados. Dos 3.125 artigos examinados para elegibilidade, 3.090 foram excluídos baseados no título ou no resumo. Os textos completos de 168 estudos potencialmente elegíveis foram avaliados. Destes, 23 cumpriram os critérios e foram incluídos na revisão (Figura 1). Os artigos foram solicitados via Biblioteca Central da Pontifícia Universidade Católica do Rio Grande do Sul, que possui acesso aos periódicos solicitados por meio do Portal Capes ou por assinatura direta.

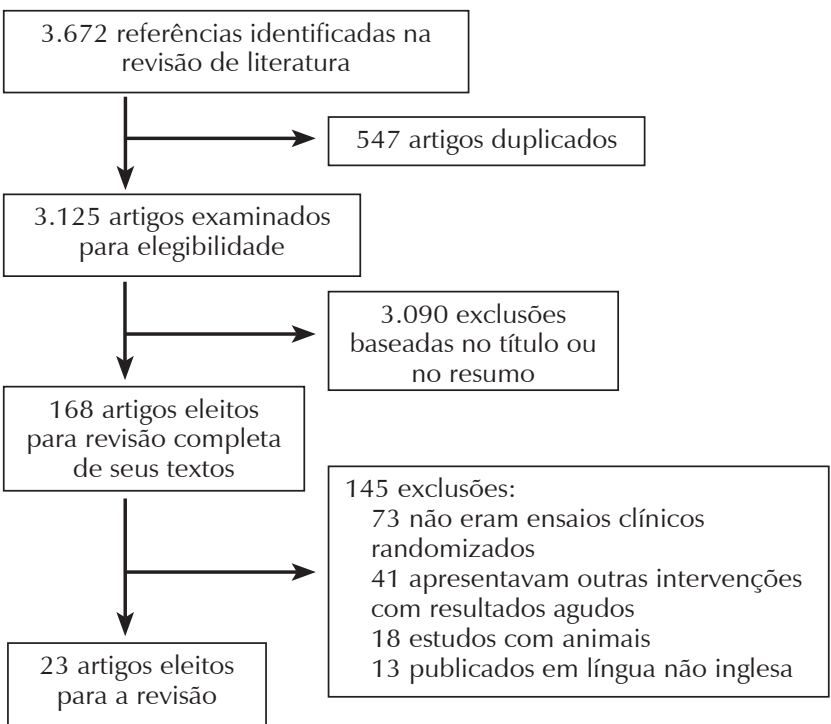

Figura 1. Intervenção do exercício na doença cardiovascular em idosos: resultados da busca na literatura, 2005 a 2016.

\section{CONTEÚDO DA REVISÃO}

Esta revisão foi focada nos desfechos clinicamente relevantes, medidos por variáveis fisiológicas e psicológicas associadas a progressão e complicações da DCV. Desfechos primários: 1) aptidão física: capaci- 
dade aeróbia, força muscular; 2) qualidade de vida relacionada a saúde (através de medidas bem estabelecidas com instrumentos confiáveis e validados); 3) dimensões cardiovasculares: índice de variabilidade de frequência cardíaca (VFC); rigidez arterial; média do intervalo das ondas R-R; velocidade de onda de pulso arterial; 4) medidas nutricionais: composição corporal (gordura visceral, circunferência da cintura) e índice de massa corporal; 5) depressão; 6) inflamação sistêmica: interleucina-6 (IL-6) e Proteína C Reativa (PCR).
Desfechos secundários: 1) lipídios sanguíneos: colesterol total, lipoproteína de densidade alta, lipoproteína de densidade baixa e os triglicerídeos. 2) progressão de DCV: nefropatia (proteinúria $>300 \mathrm{mg} /$ dia ou albuminúria $>30 \mathrm{mg} / 24$ horas) e hipertrofia do ventrículo esquerdo ao eletrocardiograma ou ao ecocardiograma.

Os resultados encontrados foram avaliados para verificar o efeito dos estudos em dois segmentos distintos: a utilização da metodologia adequada e os resultados finais encontrados em cada estudo.

Tabela 1. Descrição dos 23 estudos selecionados sobre as intervenções do exercício na doença cardiovascular em pacientes idosos.

\begin{tabular}{|c|c|c|c|c|c|}
\hline Autor/Ano & Grupos - n & Intervenção & Tempo & Estágio DCV & Resultados Primários \\
\hline El-Kader et al. 2013 [43] & $\begin{array}{l}A M-25 \\
A L-25\end{array}$ & $\begin{array}{l}\text { Aeróbico moderado } \\
\text { Aeróbico leve }\end{array}$ & $3 \mathrm{~m}$ & Sem sintomas & $\begin{array}{l}\text { Obesos diabéticos II com melhores } \\
\text { respostas das citocinas inflamatórias } \\
\text { ao treino moderado }\end{array}$ \\
\hline Bo et al. 2015 [32] & $\begin{array}{l}\mathrm{Gl}-25 \\
\mathrm{GC}-25\end{array}$ & Exercício supervisionado & 12 sem & Pós- revascularização & $\begin{array}{l}\text { Teste } 6 \text { minutos } \\
\text { Distância percorrida na esteira }\end{array}$ \\
\hline Cesa et al. 2015 [33] & $\begin{array}{l}\mathrm{Gl}-10 \\
\mathrm{GC}-9\end{array}$ & $\begin{array}{l}150 \mathrm{~min} / \mathrm{semana} \\
\text { Conselhos gerais de saúde }\end{array}$ & $\begin{array}{l}27 \text { sem } \\
\text { ou } 6 \mathrm{~m}\end{array}$ & Prevenção DCV & $\begin{array}{l}\text { Atividade física como prevenção de } \\
\text { custo baixo para DCV }\end{array}$ \\
\hline Chrysohoou et al. 2015 [34] & $\begin{array}{l}\text { GTE }-33 \\
\text { GC }-39\end{array}$ & $\begin{array}{l}\text { Treinamento de alta } \\
\text { intensidade }\end{array}$ & 12 sem & Falha cardíaca congestiva & $\begin{array}{l}\text { Dilatação da aorta } \\
\text { Pressão arterial sistólica }\end{array}$ \\
\hline Fakhry et al. 2015 [46] & $\begin{array}{c}\text { GEnR + EX - } 106 \\
G+\text { EX }-106\end{array}$ & $\begin{array}{l}\text { Funcionamento físico, dor } \\
\text { corporal e percepção geral } \\
\text { da saúde }\end{array}$ & $12 \mathrm{~m}$ & Revascularização endovascular & Distância caminhada \\
\hline Gencer et al. 2016 [41] & $\begin{array}{c}\text { GHardCHD - } 559 \\
\text { GCHD - } 884 \\
\text { GCVD }-1.106\end{array}$ & Níveis de resistina & 10.1 anos & Taxa de risco de resistina & $\begin{array}{l}\text { Associação entre resistina e DCV } \\
\text { em idosos }\end{array}$ \\
\hline Jakubseviciene et al. 2014 [31] & $\begin{array}{l}\text { Gl }-57 \\
\text { GC }-60\end{array}$ & $\begin{array}{l}\text { By-pass }+ \text { ex. supervisionado } \\
\text { By-pass }+ \text { ex. sem supervisão }\end{array}$ & $6 \mathrm{~m}$ & By-pass & $\begin{array}{l}\text { Melhorar o fluxo sanguíneo periférico, } \\
\text { SF-36 }\end{array}$ \\
\hline Jung et al. 2015 [35] & $\begin{array}{c}\text { GP }-38 \\
\text { G2.5 }-40 \\
\text { G5.0 }-43 \\
\text { G10.0 - 37 }\end{array}$ & DA - $1.229+$ exercício & 12 sem & Diabetes mellitus & Redução HbA1c \\
\hline Lakoski et al. 2015 [26] & $\begin{array}{c}\mathrm{Gl}-9 \\
\mathrm{GC}-10\end{array}$ & Treinamento + anticoagulante & $3 \mathrm{~m}$ & Diabetes mellitus & $\begin{array}{l}\text { Controle peso, aumento } \mathrm{VO}_{2} \mathrm{e} \\
\text { atividade física }\end{array}$ \\
\hline Li et al. 2015 [36] & $\begin{array}{l}\mathrm{Gl}-32 \\
\mathrm{GC}-29\end{array}$ & $\begin{array}{l}\text { Protocolo de exercício de } \\
\text { baixa intensidade em casa }\end{array}$ & 12 sem & $\begin{array}{l}\text { Fração de ejeção ventricular } \\
\text { esquerda }\end{array}$ & SF-36, Senior fitness test \\
\hline McDermott et al. 2013 [44] & $\begin{array}{l}\text { GHBW - } \\
\text { GACC }\end{array}$ & $\begin{array}{l}\text { Protocolo de treinamento } \\
\text { em casa }\end{array}$ & $6 \mathrm{~m}$ & Doença arterial periférica & $\begin{array}{l}\text { Alterações nos valores teste } 6 \text { minutos } \\
\text { caminhada }\end{array}$ \\
\hline Meid et al. 2015 [40] & $\begin{array}{c}\text { GUnder - } 1.005 \\
\text { GAppropriate - } 449\end{array}$ & Uso de medicamentos & 2.24 anos & $\begin{array}{l}\text { Subutilização de medicamentos com } \\
\text { base em critérios cardiovasculares }\end{array}$ & Consequências clínicas \\
\hline Motahari et al. 2015 [37] & $\begin{array}{l}\text { GE }-27 \\
G C-26\end{array}$ & Treinamento aeróbio & $8 \mathrm{sem}$ & Diabetes mellitus & Cintura/quadril, IMC e insulina \\
\hline Mazari et al. 2012 [38] & $\begin{array}{c}\text { GPTA - } 60 \\
\text { GSPE - } 60 \\
\text { GPTA + SPE - } 58\end{array}$ & $\begin{array}{l}\text { Treinamento aeróbico } \\
\text { supervisionado }\end{array}$ & $12 \mathrm{~m}$ & Angioplastia & $\begin{array}{l}\text { Pressão arterial no tornozelo, distância } \\
\text { caminhada e qualidade de vida }\end{array}$ \\
\hline Ortega et al. 2014 [42] & $\begin{array}{l}\text { SE }-46 \\
\text { UW }-51\end{array}$ & Cicloergômetro aeróbico & $7 \mathrm{~m}$ & Capacidade funcional & $\mathrm{VO}_{2}$ pico \\
\hline Redfield et al. 2013 [29] & $\begin{array}{l}\text { Gl }-113 \\
\text { GC }-103\end{array}$ & Citrato de sildenafila & 24 sem & Falha cardíaca & $\begin{array}{l}\mathrm{VO}_{2} \text { máximo e Teste } 6 \text { minutos de } \\
\text { caminhada }\end{array}$ \\
\hline Santalauria et al. 2013 [30] & $\begin{array}{l}\mathrm{Gl}-146 \\
\mathrm{GC}-146\end{array}$ & $\begin{array}{l}\text { Exercício aeróbico } \\
75-90 \% \text { da FC máx. }\end{array}$ & 10 sem & Infarto miocárdio & Reincidência \\
\hline Schneider et al 2011 [27] & $\begin{array}{l}\mathrm{Gl}-30 \\
\mathrm{GC}-30\end{array}$ & Exercício aeróbico & 24 sem & Diabetes mellitus e depressão & $\begin{array}{l}\text { Viabilidade e aceitação da } \\
\text { intervenção do exercício }\end{array}$ \\
\hline Taylor et al. 2014 [25] & $\begin{array}{l}\text { GHigh - } 11 \\
\text { GMod - } 10\end{array}$ & $\begin{array}{l}\text { Exercícios de resistência e } \\
\text { força }\end{array}$ & 3 meses & Diabetes mellitus & Força e resistência muscular \\
\hline Taylor-Piliae et al. 2014 [39] & $\begin{array}{l}\text { GPropPaga - } 31 \\
\text { GNPaga - } 114\end{array}$ & Recrutamento e retenção & 3 anos & Acidente vascular & $\begin{array}{l}\text { Aderência ao treinamento pós evento } \\
\text { cardíaco }\end{array}$ \\
\hline Tousignant et al. 2014 [45] & $\begin{array}{l}\text { GTele - } 120 \\
\text { GCasa - } 120\end{array}$ & Tai-Chi & 8 semanas & Reabilitação cardíaca & Tele reabilitação \\
\hline Winter et al. 2012 [28] & $\begin{array}{l}\mathrm{Gl}-28 \\
\mathrm{GC}-26\end{array}$ & Exercício aeróbico & 10 semanas & Ventrículo direito sistêmico & $\mathrm{VO}_{2}$ pico e PAS repouso \\
\hline
\end{tabular}

$\mathrm{AM}$ - aeróbio moderado; $\mathrm{AL}$ - aeróbio leve; GI - grupo intervenção; GC - grupo controle; GTE - exercise training group; GEnR - endovascular revascularization + supervised exercise GHardCHD - hard cardiovascular heart disease group; GCHD - cardiovascular heart disease; GCVD - cardiovascular event group; GP - grupo placebo; G2,5 - grupo 2,5mg DA1229; G5 - grupo 5mg DA-1229; G10 - grupo 10mg DA-1229; GHBW - home based walking group; GACC - attention control condition group; GSPE - supervised exercise program; GPTA + SPE - percutaneous transluminal angioplaty + supervised exercise program; SE - supervised exercise; UW - unsupervised walking; GHigh - high intensity group; GMod - moderate intensity group; GPropPaga - grupo propaganda paga; GNPaga - grupo propaganda não paga; GTele - grupo tele-reabilitação e GCasa - grupo treinamento em casa. 
Na Tabela 1 estão descritos os estudos em suas características metodológicas, apresentando o tamanho amostral, o tipo e duração da intervenção, o estágio de DCV, as principais medidas e os resultados finais propostos e encontrados em cada estudo. Vinte e três estudos randomizaram 7531 participantes que foram identificados e selecionados por este estudo. $\mathrm{O}$ número de participantes foi usualmente pequeno, variando entre 13 e 3044 sujeitos, sendo o último um estudo multicêntrico. Em quatro estudos (16,66\%), o tamanho da amostra foi menor do que 50 participantes [23-26]. Somente 13 estudos utilizaram grupos controle [23,26-37] adicionados a um grupo com DCV. A maioria das intervenções durou entre oito e 12 semanas; cinco ensaios clínicos duraram pelo menos um ano [24,38-41]. Do total de 23 artigos selecionados, 15 foram publicados a partir de 2015 .

Os riscos de viés, decorrentes à coleta cega dos dados, e os resultados incompletos, foram avaliados dentro da metodologia incluída em todos os estudos.

As deficiências detectadas com maior frequência estão relacionadas ao processo de randomização e a sequência de alocação da amostra. Em todos os estudos o processo de randomização foi descrito e em 10 ECR a sequência de alocação foi descrita [25-30,33,35,37,42]. O processo de cegamento dos participantes, cuidadores e avaliadores foi descrito em oito estudos [11,18,26,27,32,38,41,43]. Nenhum dos estudos apresentou a descrição dos processos de cegamento dos resultados.

Quanto às taxas de abandono, foram relatadas por 11 entre os 23 estudos apresentaram dados sobre as taxas de abandono [25,28,31,33-35,37-39,41,42]. A adesão às intervenções foi reportada em oito ECR, nos quais foi computado o número total de sessões realizadas no total previsto apresentados por porcentagem [25,27$30,33,35,36]$.

O cálculo do tamanho amostral não foi apresentado consistentemente nos artigos, tornando difícil a interpretação dos achados devido ao poder insuficiente em seis estudos [32,40,43-46]. A intervenção foi baseada em exercícios aeróbios em sete estudos $[25,28,30,33,36,37,43]$ com duração entre 30 e 75 minutos por sessão com intensidades entre 50 e $85 \%$ do $\mathrm{VO}_{2}$ máximo e entre 65 e $75 \%$ da frequência cardíaca máxima (FCM). Os exercícios de resistência foram utilizados em 22 ECR e em apenas dois foram utilizados os meios de comunicação para verificar a permanência de pacientes com DCV nos processos de treinamento físico $[11,44]$. Exercícios resistidos foram utilizados em quatro ECR $[25,30,34,43]$ e dois estudos combinaram exercícios aeróbicos e anaeróbicos $[30,43]$. Um estudo verificou a utilização adequada de medicamento [40] e um estudo verificou a aderência dos pacientes póscirurgia de revascularização a programas de exercícios físicos regulares [39]. Outro estudo desenvolveu um protocolo de tele atendimento dos pacientes com a prática de Tai-chi [45]. As intervenções mais utilizadas foram com o exercício físico supervisionado, e todos os estudos mostraram melhoras no condicionamento físico dos participantes.

\section{Resultados das variáveis pesquisadas}

Qualidade de vida relacionada à saúde. O 36item Short Form Health Survey (SF-36) [18,28,40,43] foi o instrumento mais utilizado. Os resultados encontrados nos oito domínios (funcionamento físico, desenvolvimento físico, dor corporal, saúde geral, percepção, vitalidade, funcionamento social, função emocional e saúde mental) que compõem o teste apresentaram aumento significativo em três estudos $[18,28,40]$ e apenas um estudo relatou melhora significativa em cinco domínios (saúde geral, vitalidade, funcionamento social, função emocional e saúde mental) [43]. Em um estudo foi utilizado o questionário EuroQol5 para avaliar a qualidade de vida de pacientes em prevenção secundária de reabilitação cardíaca, apresentando melhora significativa nos cinco domínios (mobilidade, cuidados pessoais, atividades habituais, dor/mal estar e ansiedade/depressão) no primeiro, quarto e décimo segundo meses após o evento cardiovascular [30]. No estudo de Mazari et al. [38] foi utilizado o questionário específico para portadores de doenças vasculares (Vascular Quality of Life Survey - VascuQol) que encontrou melhora em $71 \%$ dos pacientes com angioplastia transluminal percutânea que participavam do programa de exercícios supervisionados, foram acompanhados durante um ano e apresentaram melhora clínica sustentada, mas sem vantagem significativa na qualidade de vida.

Capacidades físicas. As medidas de capacidades físicas, como o consumo de oxigênio de pico, obtiveram incrementos entre 15 e $45 \%$ em quatro estudos $[26,28,29,42]$. A frequência cardíaca de repouso apresentou redução significativa em dois estudos $[21,30]$ e a FCM apresentou aumento ao final do período de treinamento em quatro intervenções aeróbicas [26,28,29,42]. Em média o treinamento do componente aeróbio durou de 10 semanas [18] a 07 meses [30]. A pressão arterial (sistólica e diastólica) apresentou redução quando em repouso $[21,30]$ e aumento quando avaliada a pressão arterial máxima ao final das sessões de treinamento [21]. 
Variabilidade da frequência cardíaca. Oito estudos avaliaram o efeito do exercício na VFC $[25,26,28$, $29,33,42,44,46]$. Dois estudos apresentam a relação da redução da VFC com o aumento da distância caminhada $[29,46]$ e outros três estudos a relação da diminuição da VFC com o aumento do $\mathrm{VO}_{2}$ máximo. [26,29,42].

Perfil lipídico. Alguns estudos apresentaram o perfil lipídico [26-28,33,37,38,44] como medida de resultado e encontraram diferenças na magnitude da redução das lipoproteínas a partir do modelo de intervenção do exercício. Os modelos de treinamento com durações superiores a 12 semanas apresentaram reduções significativas no LDL-c (colesterol ligado à lipoproteína de baixa densidade) e triglicerídeos, e aumento no HDL-c (colesterol ligado à lipoproteína de alta densidade). Ortega et al. [42] utilizaram o exercício supervisionado no processo primário da recuperação de DCV, verificando uma relação do aumento progressivo de carga dos exercícios e a diminuição de colesterol total, triglicerídeos, LDL-c e pressão arterial. Um estudo utilizou a técnica de Tai-Chi como intervenção em sessões que ocorreram por tele reabilitação [39], e os indivíduos apresentaram melhoras no perfil lipídico e no índice de massa corporal (IMC).

Marcadores inflamatórios. Os marcadores inflamatórios como a PCR e as citocinas IL-6 e fator de necrose tumoral (TNF)- $\alpha$ foram avaliados em oito estudos $[25,27,28,34,35,39,41,43]$. A maior parte destes teve como objetivo avaliar a resposta do diabetes no desenvolvimento de alterações vasculares. No estudo de Abd El-Kader et al. [43] foi apresentada diminuição significativa de leptina, IL-2, IL-4, IL-6 e TNF- $\alpha$ após treinamento aeróbico de intensidade leve ou moderada. Resposta similar foi encontrada nos estudos de Jung et al. [35] no grupo que realizou exercícios físicos supervisionados associados a dieta programada em indivíduos com diabetes tipo II. Gencer et al. [41] investigaram indivíduos entre 70 e 79 anos que tiveram doença arterial coronariana ou derrame. Foi identificado aumento da disponibilidade de resistina e melhoras significativas nos níveis plasmáticos de PCR num programa de treinamento que se estendeu por 10 anos. Os demais estudos não apresentaram resultados com melhoras significativas nos valores da citocinas avaliadas $[25,27,28,34,39]$.

Força muscular. Dezesseis ECR apresentaram como intervenção o treinamento de resistência [25-28, 30-32,34-38,41-45] ou treinamento aeróbico e de resistência [25]. A medida de força muscular representa um dos resultados medidos, e em três ECR [31,32,35] foi associada à intervenção no formato de exercício físico supervisionado por um profissional da área da saúde. Um ECR apresentou as vantagens da utilização do Tai Chi Chuan [45] como método de intervenção na melhoria da força muscular. O acompanhamento do desenvolvimento da força foi realizado em três ECR [25,34,41]. No estudo de Taylor et al. [37], o modelo de exercício em treinamento intenso apresentou as melhores respostas em relação ao moderado. Lakoski et al. [46] relataram que a diminuição do IMC e do peso corporal total influenciaram o tempo de execução dos exercícios. Santaularia et al. [30] verificaram a diminuição da circunferência abdominal e o controle adequado dos sintomas depressivos como fatores para a melhora na condição de força muscular como prevenção secundária à reabilitação cardíaca. Em outro estudo, Chrysohoon et al. [45] combinaram exercício aeróbico e de força muscular e obtiveram melhora significativa na força muscular e no $\mathrm{VO}_{2}$ pico, sendo que a força muscular obteve os melhores escores de incremento. Na mesma direção com indivíduos idosos, Li et al. [25] utilizaram um protocolo de exercícios que envolviam 14 articulações e caminhadas realizados em suas casas, que apresentaram melhoras significativas nos escores do Senior Fitness Test. A força necessária para a manutenção da postura e do equilíbrio corporal foi avaliada por Tousignant et al. [45], utilizando técnicas utilizadas pelo Tai Chi Chuan como modelo de exercício, e apresentaram incremento nas capacidades de mobilidade e equilíbrio dos participantes.

Composição corporal. Em nove ECR foram analisados os efeitos do exercício na composição corporal. Em quatro estudos foram avaliados pacientes com diabete mellitus [25-27,37]. Em cinco ECR, os objetivos eram a prevenção de DCV [33], intervenções cirúrgicas para melhorar a revascularização [40] e determinação da influência dos níveis de resistina no desenvolvimento de DCV [46]; e dois estudos avaliaram o resultado da angioplastia e da relação da fração de ejeção do ventrículo esquerdo $[15,44]$ na composição corporal dos avaliados. No estudo de Lakosky et al. [46], os indivíduos com tromboembolismo melhoraram seus tempos de exercício e diminuíram o IMC e o peso corporal após três meses de treinamento. No entanto, Meid et al. [40] avaliaram o abuso na utilização de medicamentos como um fator para o aumento de IMC e da fragilidade em idosos.

Estágio da $D C V$. Do total dos ECR selecionados, cinco estudos incluíram indivíduos com diabetes [25-27,35,37], três apresentaram indivíduos em recuperação de procedimento cirúrgico de revascularização $[32,45,46]$ e em dois ECR os indivíduos apresentavam falha cardíaca [29,34]. Lakosky 
et al. avaliaram pacientes com tromboembolismo venoso, trombose profunda e embolia pulmonar após o treinamento de três meses com intensidades em torno de $70 \%$ da FCM. Foram verificados diminuição do IMC e do peso corporal total e aumento do tempo de execução do exercício. Em outro estudo, Schneider et al. [27] avaliaram 60 mulheres com controle inadequado da diabetes mellitus do tipo II, com risco de desenvolvimento de doenças cardiovasculares, adicionando aos cuidados habituais um programa de exercícios durante 24 semanas, demonstrando melhora na resposta da hemoglobina glicosada e nos escores de depressão. Dois estudos avaliaram a resposta do exercício físico realizado em três sessões por semana (duas no ambiente hospitalar e uma em casa) na recuperação de 50 pacientes que realizaram angioplastia transluminal percutânea $[32,46]$. Os estudos encontraram como resposta primária uma evolução significativa nos valores do teste de seis minutos de caminhada e, como desfecho secundário, melhora na distância máxima caminhada na esteira, na caminhada livre de dores, no índice tornozelo-braquial, no volume de pulso avaliado na perna e no resultado do ultrassom para angioplastia transluminal percutânea.

Progressão da DCV. O efeito do exercício aeróbico $[11,15,18,27,30,46]$ ou treinamento resistido [32] na progressão da DCV foi medido em quatro estudos. Um declínio suave foi detectado nos índices de LDL-c [46]. Para avaliar a reabilitação cardíaca na prevenção secundária, foram recrutados pacientes com isquemia do miocárdio durante o primeiro ano de suas recuperações, para que, além dos cuidados médicos inserissem um programa de exercício supervisionado por 10 semanas para verificar as readmissões [30]. Com objetivos similares, Fahkry et al. [46] encontraram um aumento na distância máxima caminhada em 1.237 metros e na distância caminhada livre de dor em 1.120 metros em pacientes com revascularização endovascular e exercícios supervisionados durante 12 meses de terapia combinada. O estudo de Bo et al. [32] revelou um aumento na distância máxima caminhada em 251 metros e de 66 metros no teste de caminhada de seis minutos em um período de três meses para pacientes que apresentaram angioplastia no último ano.

Depressão. A depressão foi avaliada em três estudos que utilizavam exercícios aeróbios [15,28,44]. Os estudos apresentam o exercício físico moderado e regular como mecanismo capaz de reverter os escores de depressão, porém somente um dos ECR apresentados nesta revisão conseguiu apresentar os benefícios do exercício físico regular nos níveis de depressão. McDermott et al. [44] encontraram melhoras significativas na condição de depressão associadas ao incremento da distância máxima caminhada e ao aumento da velocidade de deslocamento sem ocorrer claudicação.

\section{DISCUSSÃO}

Esta revisão sistemática reuniu evidências do efeito das boas práticas relacionadas à informação, inserção e principalmente a adesão de indivíduos com DCV a programas de exercício aeróbio com reflexos positivos no condicionamento físico, na força muscular e na qualidade de vida nas diferentes fases do envelhecimento. As evidências são fracas e heterogêneas para indicar o exercício físico como fator de mudança na condição de saúde dos idosos que apresentavam sintomas iniciais de DCV. Apesar deste quadro, alguns resultados significativos foram obtidos quanto às boas práticas de exercícios físicos. Por exemplo, TaylorPiliae et al. [39] e Tousignant et al. [45] determinaram que a melhor estratégia de recrutamento e seleção está relacionada ao aviso e propagandas pagas na mídia em geral para a manutenção da comunicação utilizando inclusive metodologias de tele-reabilitação para o acompanhamento do treinamento físico. As estratégias à distância estão modificando e atingem um público maior de indivíduos com alterações cardíacas. Li et al. [36] e Meid et al. [40] utilizaram o procedimento padrão, com protocolos de exercícios realizados em casa e encontraram resultados de melhora significativa na função física do senior fitness test.

O baixo condicionamento físico é independentemente um preditor de mortalidade em muitas populações ao desenvolverem doenças crônicas. O treinamento físico tem a capacidade de melhorar o condicionamento funcional dos indivíduos, podendo hipoteticamente melhorar os índices de sobrevivência com qualidade. Entretanto não existem ECR até o momento que sustentam essa hipótese [33]. Os ECR utilizam populações mais jovens para ajustar os procedimentos de prevenção de DCV. Então avaliar a capacidade funcional como um fator prognóstico deve ser utilizado desde cedo como indicador de doenças coronarianas. Os ECR revelaram resultados positivos em inumeras variáveis fisiológicas investigadas. Gencer et al. [41], Taylor et al. [25] e Ortega et al. [42] avaliaram os eventos relacionados a doença cardiovascular e encontraram uma diminuição nos fatores de desenvolvimento de doença metabólica tais como: IMC, glicose plasmática em jejum, tecido adiposo abdominal visceral e subcutâneo, leptina, adiponectina e insulina e de inflamação sistêmica como: PCR, IL-6, TNF- $\alpha$. 
O condicionamento físico, representado pelo controle da FC apresenta um fator interessante e a associação entre DCV e baixa VFC é consistente através da avaliação de indicadores dos multiplos estágios da doença que incluem idade avançada, obesidade, diabetes, estilo de vida sedentário, altos níveis de insulina, pressão arterial sistólica elevada [30,34, $43,45,47]$ e a diminuição da PCR e das lipoproteínas (LDL-c, colesterol total e triglicerideos) $[33,46]$. O impacto da VFC na mortalidade pode ser explicado pelo balanço parassimpático/simpático com a superativação do sistema simpático em indivíduos com baixa VFC que aumenta as possibilidades de desenvolvimento de arritmias malignas [47].

$\mathrm{O}$ indicador de qualidade no treinamento mais utilizado foi o teste de caminhada de seis minutos e Jakubseviciene et al. [31], Bo et al. [32], Chrysohoon et al. [34], McDermott et al. [44] e Fakhry et al. [46] avaliaram pacientes pós evento cardiovascular cirúrgico e foi observada uma melhora significativa na caminhada livre de dor investigada durante o teste de esforço e os efeitos positivos do HIIT (high intense intermitent training) na função ventricular esquerda.

Apesar dos resultados encontrados, esta revisão sistemática possui algumas limitações, principalmente metodológicas [47]. Durante a preparação desta revisão, um número grande de ensaios clínicos foram excluídos devido a falta de randomização dos participantes. A ocultação da alocação parece ser um item importante. O processo de cegamento quando inadequado leva ao aumento do risco de interferência do viés de diversas maneiras e em algumas vezes levando os resultados a subversões deliberadas (em sua maioria bem intencionadas ou como resultado de ações subconscientes) apresentando ensaios clínicos com metodos de cegamento inadequados ou obscuros. Outra limitação consiste no fato de terem sido incluídos apenas artigos no idioma inglês, o que pode ter omitido estudos importamtes com resultados descritos em outro idioma. Além disso a maioria dos ECR não analizou de acordo com o principio intention-totreat, aumentando o risco de seleção de viés. Também ocorrem deficiências ao reportar as informações motodológicas e seus achados como o índice de abandono, aderência a intervenção e descrição do grupo controle. Os leitores da presente revisão devem ser alertados de que é possivel que um ensaio clínico pode ser classificado como sendo de baixa qualidade, e que realmente tenha, pois os dados e/ou informações não foram descritos. Apesar da disponibilidade de orientações destinadas a padronizar o relato de ensaios clínicos, as publicações, muitas vezes omitem detalhes metodológicos essenciais [25,27-30,33,35,36].

\section{CONSIDERAÇÕES FINAIS}

Além de problemas metodológicos, de comunicação e de troca de informações com a literatura atualizada sobre os efeitos do exercício sobre a saúde dos individuos em envelhecimento, detectamos a escassez de ensaios incluindo pacientes em estágios iniciais de DCV. Entretanto, apesar da falta de evidências baseadas em ECR sobre o efeito do exercício na mortalidade, os efeitos documentados do exercício no condicionamento físico, na força, na qualidade de vida e na condição cardiovascular são suficientes para apoiar a recomendação de atividade física de intensidade moderada (entre 60 e $75 \%$ da FCM) para pacientes com DCV em qualquer estágio de desenvolvimento da doença. As intervenções que utilizam o exercício precisam ser progressivamente incluídas nos regimes dos centros de tratamento cardiovascular, embora o melhor protocolo de exercícios para pacientes com DCV ainda não esteja completamente estabelecido.

\section{NOTAS}

Apoio financeiro

Este estudo teve apoio financeiro do Conselho Nacional de Desenvolvimento Científico e Tecnológico (CNPq) e da Fundação de Amparo à Pesquisa do Estado do Rio Grande do Sul (FAPERGS).

\section{Conflito de Interesses}

Os autores declaram não haver conflitos de interesses relevantes ao conteúdo deste estudo. Eles informam ter tido acesso a todos os dados obtidos e assumem completa responsabilidade pela integridade dos resultados.

\section{REFERÊNCIAS}

1. Partridge L. The new biology of ageing. Philos Trans R Soc Lond B Biol Sci. 2010 Jan 12;365(1537):147-54. https:// doi.org/10.1098/rstb.2009.0222

2. Flicker L. Cardiovascular risk factors, cerebrovascular disease burden, and healthy brain aging. Clin Geriatr Med. 2010 Feb;26(1):17-27. https://doi.org/10.1016/j.cger.2009.12.005

3. Huffman DM. Exercise as a calorie restriction mimetic: implications for improving healthy aging and longevity. Interdiscip Top Gerontol. 2010;37:157-74. https://doi.org/10.1159/000320000 
4. Ji LL, Dickman JR, Kang C, Koenig R. Exercise-induced hormesis may help healthy aging. Dose Response. 2010 Jan 28;8(1):73-9. https://doi.org/10.2203/dose-response.09-048.Ji

5. Muscari A, Giannoni C, Pierpaoli L, Berzigotti A, Maietta P, Foschi E, Ravaioli C, Poggiopollini G, Bianchi G, Magalotti D, Tentoni C, Zoli M. Chronic endurance exercise training prevents aging-related cognitive decline in healthy older adults: a randomized controlled trial. Int J Geriatr Psychiatry. 2010 Oct;25(10):1055-64. https://doi.org/10.1002/ gps. 2462

6. Rolland Y, Abellan van Kan G, Vellas B. Healthy brain aging: role of exercise and physical activity. Clin Geriatr Med. 2010 Feb;26(1):75-87. https://doi.org/10.1016/j.cger.2009.11.002

7. Davis JW, Chung R, Juarez DT. Prevalence of comorbid conditions with aging among patients with diabetes and cardiovascular disease. Int J Geriatr Psychiatry. 2010 Oct;25(10):1055-64.

8. Partridge L. Diet and healthy aging. N Engl J Med. 2012 Dec 27;367(26):2550-1. https://doi.org/10.1056/NEJMcibr1210447

9. Santos-Parker JR, LaRocca TJ, Seals DR. Aerobic exercise and other healthy lifestyle factors that influence vascular aging. Adv Physiol Educ. 2014 Dec;38(4):296-307. https://doi.org/10.1152/advan.00088.2014

10. van Empel VP, Kaye DM, Borlaug BA. Effects of healthy aging on the cardiopulmonary hemodynamic response to exercise. Am J Cardiol. 2014 July 1;114(1):131-5. https://doi.org/10.1016/j.amjcard.2014.04.011

11. Lesniewski LA, Zigler ML, Durrant JR, Nowlan MJ, Folian BJ, Donato AJ, Seals DR. Aging compounds western dietassociated large artery endothelial dysfunction in mice: prevention by voluntary aerobic exercise. Exp Gerontol. 2013 Nov;48(11):1218-25. https://doi.org/10.1016/j.exger.2013.08.001

12. Willcox DC, Scapagnini G, Willcox BJ. Healthy aging diets other than the Mediterranean: a focus on the Okinawan diet. Mech Ageing Dev. 2014 Mar-Apr;136-137:148-62. https://doi.org/10.1016/j.mad.2014.01.002

13. Bolduc V, Thorin-Trescases N, Thorin E. Endothelium-dependent control of cerebrovascular functions through age: exercise for healthy cerebrovascular aging. American journal of physiology Am J Physiol Heart Circ Physiol. 2013 Sept 1;305(5):H620-33. https://doi.org/10.1152/ajpheart.00624.2012

14. Joyner MJ. Buying into healthy blood vessels: exercise and aging. J Appl Physiol (1985). 2014 Sept 1;117(5):421-2.

15. Wister AV. Population pressures, system-level inertia and healthy aging policy revisited. Healthc Pap. 2011;11(1):41-5; discussion 86-91. https://doi.org/10.12927/hcpap.2011.22251

16. Bori Z, Zhao Z, Koltai E, Fatouros IG, Jamurtas AZ, Douroudos II, Terzis G, Chatzinikolaou A, Sovatzidis A, Draganidis D, Boldogh I, Radak Z. The effects of aging, physical training, and a single bout of exercise on mitochondrial protein expression in human skeletal muscle. Exp Gerontol. 2012 June;47(6):417-24. https://doi.org/10.1016/j.exger.2012.03.004

17. North BJ, Sinclair DA. The intersection between aging and cardiovascular disease. Circ Res. 2012 Apr 13;110(8): 1097-108. https://doi.org/10.1161/CIRCRESAHA.111.246876

18. Gast U, Belavý DL, Armbrecht G, Kusy K, Lexy H, Rawer R, Rittweger J, Winwood K, Zieliński J, Felsenberg D. Bone density and neuromuscular function in older competitive athletes depend on running distance. Osteoporos Int. 2013 July;24(7):2033-42. https://doi.org/10.1007/s00198-012-2234-0

19. Heo J, Culp B, Yamada N, Won Y. Promoting successful aging through competitive sports participation: insights from older adults. Qual Health Res. 2013 Jan;23(1):105-13. https://doi.org/10.1177/1049732312457247

20. Moher D, Liberati A, Tetzlaff J, Altman DG; PRISMA Group. Reprint--preferred reporting items for systematic reviews and meta-analyses: the PRISMA statement. Phys Ther. 2009 Sept;89(9):873-80.

21. Moher D, Liberati A, Tetzlaff J, Altman DG; PRISMA Group. Preferred reporting items for systematic reviews and meta-analyses: the PRISMA statement. J Clin Epidemiol. 2009 Oct;62(10):1006-12. https://doi.org/10.1016/j. jclinepi.2009.06.005

22. Higgins JP, Altman DG, Gøtzsche PC, Jüni P, Moher D, Oxman AD, Savovic J, Schulz KF, Weeks L, Sterne JA; Cochrane Bias Methods Group; Cochrane Statistical Methods Group. The Cochrane Collaboration's tool for assessing risk of bias in randomised trials. BMJ. 2011 Oct 18;343:d5928. https://doi.org/10.1136/bmj.d5928

23. de Souto Araujo ZT, de Miranda Silva Nogueira PA, Cabral EE, de Paula Dos Santos L, da Silva IS, Ferreira GM. Effectiveness of low-intensity aquatic exercise on COPD: a randomized clinical trial. Respir Med. 2012 Nov;106(11):153543. https://doi.org/10.1016/j.rmed.2012.06.022

24. Fayh AP, Lopes AL, da Silva AM, Reischak-Oliveira A, Friedman R. Effects of $5 \%$ weight loss through diet or diet plus exercise on cardiovascular parameters of obese: a randomized clinical trial. Eur J Nutr. 2013 Aug;52(5):1443-50. https:// doi.org/10.1007/s00394-012-0450-1

25. Taylor JD, Fletcher JP, Mathis RA, Cade WT. Effects of moderate- versus high-intensity exercise training on physical fitness and physical function in people with type 2 diabetes: a randomized clinical trial. Phys Ther. 2014 Dec;94(12):172030. https://doi.org/10.2522/ptj.20140097

26. Lakoski SG, Savage PD, Berkman AM, Penalosa L, Crocker A, Ades PA, Kahn SR, Cushman M. The safety and efficacy of early-initiation exercise training after acute venous thromboembolism: a randomized clinical trial. J Thromb Haemost. 2015 July;13(7):1238-44. https://doi.org/10.1111/jth.12989 
27. Schneider KL, Pagoto SL, Handschin B, Panza E, Bakke S, Liu Q, Blendea M, Ockene IS, Ma Y. Design and methods for a pilot randomized clinical trial involving exercise and behavioral activation to treat comorbid type 2 diabetes and major depressive disorder. Ment Health Phys Act. 2011 June 1;4(1):13-21. https://doi.org/10.1016/j.mhpa.2011.04.001

28. Winter MM, van der Bom T, de Vries LC, Balducci A, Bouma BJ, Pieper PG, van Dijk AP, van der Plas MN, Picchio FM, Mulder BJ. Exercise training improves exercise capacity in adult patients with a systemic right ventricle: a randomized clinical trial. Eur Heart J. 2012 June;33(11):1378-85. https://doi.org/10.1093/eurheartj/ehr396

29. Redfield MM, Chen HH, Borlaug BA, Semigran MJ, Lee KL, Lewis G, LeWinter MM, Rouleau JL, Bull DA, Mann DL, Deswal A, Stevenson LW, Givertz MM, Ofili EO, O'Connor CM, Felker GM, Goldsmith SR, Bart BA, McNulty SE, Ibarra JC, Lin G, Oh JK, Patel MR, Kim RJ, Tracy RP, Velazquez EJ, Anstrom KJ, Hernandez AF, Mascette AM, Braunwald E; RELAX Trial. Effect of phosphodiesterase-5 inhibition on exercise capacity and clinical status in heart failure with preserved ejection fraction: a randomized clinical trial. JAMA. 2013 Mar 27;309(12):1268-77. https://doi. org/10.1001/jama.2013.2024

30. Santaularia N, Caminal J, Arnau A, Perramon M, Montesinos J, Trapé J, Abenoza-Guardiola M, Guiteras-Val P, Jaarsma T. Randomized clinical trial to evaluate the effect of a supervised exercise training program on readmissions in patients with myocardial ischemia: a study protocol. BMC Cardiovasc Disord. 2013 Apr 25;13:32. https://doi.org/10.1186/14712261-13-32

31. Jakubseviciene E, Vasiliauskas D, Velicka L, Kubilius R, Milinaviciene E, Vencloviene J. Effectiveness of a new exercise program after lower limb arterial blood flow surgery in patients with peripheral arterial disease: a randomized clinical trial. Int J Environ Res Public Health. 2014 Aug 7;11(8):7961-76. https://doi.org/10.3390/ijerph110807961

32. Bø E, Bergland A, Stranden E, Jørgensen JJ, Sandbaek G, Grøtta OJ, Hisdal J. Effects of 12 Weeks of Supervised Exercise After Endovascular Treatment: A Randomized Clinical Trial. Physiother Res Int. 2015 Sept;20(3):147-57. https://doi. org/10.1002/pri.1608

33. Cesa CC, Barbiero SM, Petkowicz Rde O, Martins CC, Marques Rd, Andreolla AA, Pellanda LC. Effectiveness of physical exercise to reduce cardiovascular risk factors in youths: a randomized clinical trial. J Clin Med Res. 2015 May;7(5):348-55. https://doi.org/10.14740/jocmr1700w

34. Chrysohoou C, Angelis A, Tsitsinakis G, Spetsioti S, Nasis I, Tsiachris D, Rapakoulias P, Pitsavos C, Koulouris NG, Vogiatzis I, Dimitris T. Cardiovascular effects of high-intensity interval aerobic training combined with strength exercise in patients with chronic heart failure. A randomized phase III clinical trial. Int J Cardiol. 2015 Jan 20;179:269-74. https:// doi.org/10.1016/j.ijcard.2014.11.067

35. Jung CH, Park CY, Ahn KJ, Kim NH, Jang HC, Lee MK, Park JY, Chung CH, Min KW, Sung YA, Park JH, Kim SJ, Lee HJ, Park SW. A randomized, double-blind, placebo-controlled, phase II clinical trial to investigate the efficacy and safety of oral DA-1229 in patients with type 2 diabetes mellitus who have inadequate glycaemic control with diet and exercise. Diabetes Metab Res Rev. 2015 Mar;31(3):295-306. https://doi.org/10.1002/dmrr.2613

36. Li X, Xu S, Zhou L, Li R, Wang J. Home-Based Exercise in Older Adults Recently Discharged From the Hospital for Cardiovascular Disease in China: Randomized Clinical Trial. Nurs Res. 2015 July-Aug;64(4):246-55. https://doi. org/10.1097/NNR.0000000000000102

37. Motahari-Tabari N, Ahmad Shirvani M, Shirzad EAM, Yousefi-Abdolmaleki E, Teimourzadeh M. The effect of 8 weeks aerobic exercise on insulin resistance in type 2 diabetes: a randomized clinical trial. Glob J Health Sci. 2014 Aug 14;7(1):115-21. https://doi.org/10.5539/gjhs.v7n1p115

38. Mazari FA, Khan JA, Carradice D, Samuel N, Abdul Rahman MN, Gulati S, Lee HL, Mehta TA, McCollum PT, Chetter IC. Randomized clinical trial of percutaneous transluminal angioplasty, supervised exercise and combined treatment for intermittent claudication due to femoropopliteal arterial disease. Br J Surg. 2012 Jan;99(1):39-48. https://doi.org/10.1002/ bjs. 7710

39. Taylor-Piliae RE, Boros D, Coull BM. Strategies to improve recruitment and retention of older stroke survivors to a randomized clinical exercise trial. J Stroke Cerebrovasc Dis. 2014 Mar;23(3):462-8. https://doi.org/10.1016/j. jstrokecerebrovasdis.2013.03.031

40. Meid AD, Quinzler R, Freigofas J, Saum KU, Schöttker B, Holleczek B, Heider D, König HH, Brenner H, Haefeli WE. Medication Underuse in Aging Outpatients with Cardiovascular Disease: Prevalence, Determinants, and Outcomes in a Prospective Cohort Study. PLoS One. 2015 Aug 19;10(8):e0136339. https://doi.org/10.1371/journal.pone.0136339

41. Gencer B, Auer R, de Rekeneire N, Butler J, Kalogeropoulos A, Bauer DC, Kritchevsky SB, Miljkovic I, Vittinghoff E, Harris T0, Rodondi N. Association between resistin levels and cardiovascular disease events in older adults: The health, aging and body composition study. Atherosclerosis. 2016 Feb;245:181-6. https://doi.org/10.1016/j.atherosclerosis.2015.12.004

42. Ortega R, Garcia-Ortiz L, Torcal J, Echevarria P, Vargas-Machuca C, Gomez A, Salcedo F, Lekuona I, Montoya I, Grandes G; ESCAP Group. Supervised exercise for acute coronary patients in primary care: a randomized clinical trial. Fam Pract. 2014 Feb;31(1):20-9. https://doi.org/10.1093/fampra/cmt059

43. Abd El-Kader S, Gari A, Salah El-Den A. Impact of moderate versus mild aerobic exercise training on inflammatory cytokines in obese type 2 diabetic patients: a randomized clinical trial. Afr Health Sci. 2013 Dec;13(4):857-63. https:// doi.org/10.4314/ahs.v13i4.1 
44. McDermott MM, Liu K, Guralnik JM, Criqui MH, Spring B, Tian L, Domanchuk K, Ferrucci L, Lloyd-Jones D, Kibbe M, Tao H, Zhao L, Liao Y, Rejeski WJ. Home-based walking exercise intervention in peripheral artery disease: a randomized clinical trial. JAMA. 2013 July 3;310(1):57-65. https://doi.org/10.1001/jama.2013.7231

45. Tousignant M, Corriveau H, Kairy D, Berg K, Dubois MF, Gosselin S, Swartz RH, Boulanger JM, Danells C. Tai Chibased exercise program provided via telerehabilitation compared to home visits in a post-stroke population who have returned home without intensive rehabilitation: study protocol for a randomized, non-inferiority clinical trial. Trials. 2014 Jan 30;15:42. https://doi.org/10.1186/1745-6215-15-42

46. Fakhry F, Spronk S, van der Laan L, Wever JJ, Teijink JA, Hoffmann WH, Smits TM, van Brussel JP, Stultiens GN, Derom A, den Hoed PT, Ho GH, van Dijk LC, Verhofstad N, Orsini M, van Petersen A, Woltman K, Hulst I, van Sambeek MR, Rizopoulos D, Rouwet EV, Hunink MG. Endovascular Revascularization and Supervised Exercise for Peripheral Artery Disease and Intermittent Claudication: A Randomized Clinical Trial. JAMA. 2015 Nov 10;314(18):1936-44. https://doi. org/10.1001/jama.2015.14851

47. Barcellos FC, Santos IS, Umpierre D, Bohlke M, Hallal PC. Effects of exercise in the whole spectrum of chronic kidney disease: a systematic review. Clin Kidney J. 2015 Dec;8(6):753-65. https://doi.org/10.1093/ckj/sfv099 C 